\title{
Evidenzbasierte Implementierung von stationsäquivalenter Behandlung in Deutschland
}

\section{Evidence-Based Implementation of Crisis Resolution in Germany}

\author{
Autoren \\ Martin Lambert ${ }^{1}$, Anne Karow ${ }^{1}$, Jürgen Gallinat ${ }^{1}$, Arno Deister ${ }^{2}$ \\ Institute \\ 1 Klinik für Psychiatrie und Psychotherapie, Zentrum für \\ Psychosoziale Medizin, Universitätsklinikum Hamburg- \\ Eppendorf (UKE), Hamburg \\ 2 Klinikum Itzehoe, Zentrum für Psychosoziale Medizin, Itzehoe
}

\author{
Bibliografie \\ DOI http://dx.doi.org/10.1055/s-0043-100494 \\ Psychiat Prax 2017; 44: 62-64 \\ (c) Georg Thieme Verlag KG Stuttgart · New York \\ ISSN 0303-4259 \\ Korrespondenzadresse \\ Prof. Dr. Martin Lambert, Arbeitsbereich Psychosen, Klinik und \\ Poliklinik für Psychiatrie und Psychotherapie, Zentrum für \\ Psychosoziale Medizin, Universitätsklinikum Hamburg-Eppendorf, \\ Martinistraße 52, 20246 Hamburg \\ lambert@uke.de
}

2016 hat die Bundesregierung das „Gesetz zur Weiterentwicklung der Versorgung und der Vergütung für psychiatrische und psychosomatische Leistungen " (PsychVVG) beschlossen [1].

Zur Förderung der sektorenübergreifenden Versorgung wurde der $\S 39$ Absatz 1 des Fünften Buches Sozialgesetzbuch (SGB V) geändert: „Die Krankenhausbehandlung wird vollstationär, stationsäquivalent, teilstationär, vor- und nachstationär sowie ambulant erbracht."

Die stationsäquivalente Behandlung umfasst „...eine psychiatrische Behandlung im häuslichen Umfeld durch mobile ärztlich geleitete multiprofessionelle Behandlungsteams. Sie entspricht hinsichtlich der Inhalte sowie der Flexibilität und Komplexität der Behandlung einer vollstationären Behandlung. “

Im neuen §115d wurde die stationsäquivalente Behandlung genauer definiert: „Psychiatrische Krankenhäuser mit regionaler Versorgungsverpflichtung sowie Allgemeinkrankenhäuser mit selbstständigen, fachärztlich geleiteten psychiatrischen Abteilungen mit regionaler Versorgungsverpflichtung können in medizinisch geeigneten Fällen, wenn eine Indikation für eine stationäre psychiatrische Behandlung vorliegt, anstelle einer vollstationären Behandlung eine stationsäquivalente psychiatrische Behandlung im häuslichen Umfeld erbringen. “ Weiter heißt es: „... kann das Krankenhaus an der ambulanten psychiatrischen Versorgung teilnehmende Leistungserbringer oder ein anderes Krankenhaus mit der Durchführung von Teilen der Behandlung beauftragen. “

Bis 30. Juni 2017 sind 4 Punkte zu klären: „die Anforderungen an Dokumentation, Qualität der Leistungserbringung, Beauftragung teilnehmender Leistungserbringer und Grundsätze für den Abbau nicht mehr erforderlicher Betten".

Mit den $\S \S 39$ und 115d wurden die gesetzlichen Grundlagen für eine stationsäquivalente Behandlung im häuslichen Umfeld geschaffen. Diese kann in „medizinisch geeigneten Fällen, wenn eine Indikation für eine stationäre psychiatrische Behandlung vorliegt..." angewendet werden. Diese Kriterien verdeutlichen, dass es sich im Kern um eine stationsäquivalente Akutbehandlung im häuslichen Umfeld handelt.

Im Sinne der evidenzbasierten Medizin stellt sich damit die Frage, welches Behandlungsmodell zur Akutbehandlung im häuslichen Umfeld die größte Evidenz aufweist. Diese Frage ist von zentraler Bedeutung, weil

1. ein evidentes Behandlungsmodell eine größtmögliche

Effektivität und zumeist auch Effizienz aufweist,

2. es über etablierte Qualitätsindikatoren verfügt, die eine qualitätsgesicherte Implementierung, Zertifizierung, Auditierung, Leistungsdokumentation und Begutachtung erlauben,

3. es im Bereich der Akutbehandlung im häuslichen die größtmögliche Sicherheit von Klienten, Angehörigen und anderen Bezugspersonen sowie Behandlern gewährleistet.

Das Behandlungsmodell, welches eine stationsäquivalente Akutbehandlung im häuslichen Umfeld umfasst, wird am ehesten als Crisis Resolution (Team) (CRT) oder Crisis Resolution and Home Treatment (Team) (CRHTT) [2-6] bezeichnet. Nachfolgend wird der Begriff Crisis Resolution Team (CRT) verwendet.

Die Definition von CRT ist ein fachärztlich geleitetes, multiprofessionelles Team mit der Aufgabe einer zeitlich begrenzten Akutbehandlung im häuslichen Umfeld für Klienten in akuten Krankheitsphasen einer psychischen Erkrankung [2, 3, 6, 7].

Die Kernelemente von CRTs sind [3, 4, 6, 8]:

- sie arbeiten multiprofessionell,

- sie haben nur die Aufgabe der häuslichen Krisenintervention,

- sie sind einfach erreichbar, reagieren schnell auf Überweisungen und akzeptieren Überweisungen von allen Ressourcen,

- sie stehen 24 Stunden täglich an 7 Tagen der Woche zur Krisenintervention bereit, 
- sie haben eine „Gatekeeping“-Funktion für alle stationären Akutaufnahmen,

- sie behandeln Klienten unabhängig von Diagnose, Erkrankungsphase und Behandlungsstatus,

- sie bieten eine intensive Krisenintervention über einen kurzen Zeitraum,

- sie stehen in engem Kontakt mit den Klienten und anderen Bezugspersonen (etwa 1-mal pro Schicht),

- sie bieten pharmakologische, psychologische, soziale, somatische Behandlungen bzw. koordinieren diese,

- sie behandeln solange bis die Krise beendet und eine adäquate Weiterbehandlung organisiert ist,

- sie sichern die Weiterbehandlung der Klienten und

- sie stehen für erneute Kriseninterventionen bereit.

CRTs sind klinisch effektiv und effizient mit den wissenschaftlich erwiesenen Vorteilen einer Verhinderung und Verkürzung stationärer Behandlungen und einer größeren Zufriedenheit bei Klienten und Angehörigen gegenüber der stationären Akutbehandlung [5, 6]. Aus der klinischen Erfahrung sind zahlreiche weitere Vorteile bekannt [2, 3], u.a. (1) Engagement und Krisenintervention von unbehandelten Klienten, bei denen keine Eigen- oder Fremdgefährdung besteht; (2) größere Flexibilität der Interventionen (v. a. Ort und Zeit); (3) besseres Verständnis von Symptomen, Funktionsniveau, sozialen Problemen, Kultur und Bedürfnisse durch Behandlung im häuslichen Umfeld; (4) regelhafte Unterstützung, Beratung und Psychoedukation von im häuslichen Umfeld lebenden Personen (Angehörige, Partner, Kinder etc.); (5) Verminderung von Stigmatisierung durch Krisenbewältigung im eigenen Lebensumfeld; (6) Größere Stärkung der Krisenbewältigungsfähigkeiten des Klienten; (7) Reduktion von Behandlungsabbruch und Zwangseinweisungen.

Die Indikationen und Kontraindikationen hängen u. a. von der Ausgestaltung der CRTs ab. Wichtige Indikationen umfassen das Bestehen einer psychischen Erkrankung und das Vorliegen einer psychischen Krise [6]. Kontraindikationen sind zumeist relativ und beziehen sich v.a. auf die initiale Akutbehandlung, z. B. Eigen- oder Fremdgefährdungen bzw. psychiatrische Notfälle, die eine sofortige Sicherung notwendig machen oder somatische Erkrankungen, die im Krankenhaus behandelt werden müssen.

Implementierung, Zertifizierung und Auditierung von CRTs können u. a. anhand von Qualitätsindikatoren erfolgen. Diesbezüglich hat das National Institute for Health Research (NIHR) in England kürzlich die CORE Crisis Resolution Team Fidelity Scale Version 2 publiziert [6, 8].

Die Qualitätsindikatoren der CORE-Skala basieren auf 3 Quellen: (1) einem systematischen Review von quantitativen und qualitativen Studien und Leitlinien zur Implementierung von CRTs [4], (2) einem nationalen Survey bei CRT-Managern in England mit Befragungen zu Organisation und Servicestrukturen sowie deren Meinung zu prioritären Komponenten einer effektiven CRT-Behandlung und (3) von über 100 Interviews und Fokusgruppen mit CRT-„Stakeholdern“ u. a. inklusive Klienten, Angehörige und andere Bezugspersonen und Kliniker [8]. Die Skala ist für die Auditierung schon implementierter CRTs konzipiert. Entsprechend gehören hierzu ein Review von Patienten- akten und Interviews mit CRT-Managern, Teammitgliedern, Klienten und Angehörigen sowie Verantwortliche von anderen Behandlungsinstitutionen.

Die CORE-Skala hat 4 Cluster mit 39 Items, die jeweils anhand von skalierten Qualitätskriterien bewertet werden. Die 4 Cluster umfassen:

1. Überweisung und Zugang (Item 1-10),

2. Inhalt und Bereitstellung der Behandlung (Item 11-26),

3. Personal und Teamprozesse (Item 27-36),

4. Zeit und Ort der Behandlung (Item 37 -39).

Die Items der CORE-Skala stellen gewissermaßen die Kernkomponenten von CRTs dar. Darüber hinaus sind aber auch noch andere Komponenten zu berücksichtigen [2, 3].

Die besondere Herausforderung ist nun, dass einige dieser Komponenten strukturelle und prozessuale Implikationen für das gesamte Versorgungssystem haben. Hierzu gehören beispielsweise die Verankerung von CRTs als Akutbehandlungsinstitution mit Gatekeeping-Funktion für alle stationären Akutaufnahmen im Versorgungssektor oder Zusammenarbeit und Schnittstellenmanagement zu bestehenden Versorgungsinstitutionen. Hinzu kommt, dass andere evidenzbasierte ambulante Behandlungsmodelle, mit denen CRTs regelhaft zusammenarbeiten, in Deutschland noch nicht flächendeckend implementiert sind. Hierzu gehören im Bereich schwerer psychischer Erkrankungen welche die Hauptklientel von CRTs darstellen - vor allem sog. Early Intervention Services (EIS) zur Früherkennung und Frühbehandlung oder Assertive Community Treatment (ACT) zu deren langfristiger Behandlung [9]. Auch die Mitarbeit von Peers (= geschulte Gesundheitsbegleiter) in CRTs ist noch nicht verankert [9].

Zusammenfassend wurde mit den Gesetzen zur stationsäquivalenten Behandlung im häuslichen Umfeld in Deutschland wesentliche Voraussetzungen zur Verbesserung der evidenzbasierten Behandlung von Menschen mit psychischen Erkrankungen geschaffen. Damit besteht erstmals die Möglichkeit, einige zentrale Forderungen der Agenda 2020 des Bundesverbands der Angehörigen psychisch Kranker flächendeckend umzusetzen [10]. Aus Sicht der Autoren sind für die erfolgreiche Implementierung von CRTs folgende Aspekte von zentraler Bedeutung: (1) Berücksichtigung von etablierten Qualitätsindikatoren, (2) Umsetzung in enger Zusammenarbeit sektoraler und interdisziplinärer Leistungserbringer und (3) Implementierung einer Finanzierung, welche die Umsetzung von etablierten Qualitätsindikatoren ermöglicht. Langfristig ist darüber hinaus die konsequente Weiterentwicklung evidenzbasierter langfristiger ambulanter Behandlungsmodelle notwendig. 


\section{Literatur}

[1] Deutscher Bundestag. Gesetzentwurf der Bundesregierung zur Weiterentwicklung der Versorgung und der Vergütung für psychiatrische und psychosomatische Leistungen (PsychVVG).https://www.bundes gesundheitsministerium.de/fileadmin/Dateien/3_Downloads/Geset ze_und_Verordnungen/GuV/P/160803_PsychVVG_Kabinett.pdf 2016

[2] Johnson S, Needle J, Bindman JP, Thornicroft G. Crisis Resolution and Home Treatment in Mental Health. Cambridge: Cambridge University Press; 2008

[3] Smyth M, Hefferman K, Rowland A. Crisis Resolution and Home Treatment. National Institute for Mental Health England, NIMHE West Midslands; 2008: Im Internet: http://bcu.ac.uk/Download/Asset/ 50450b3b-d18c-4900-b985-5218b3b13afb (Stand: 06.01.2017)

[4] Wheeler C, Lloyd-Evans B, Churchard A et al. Implementation of the Crisis Resolution Team model in adult mental health settings: a systematic review. BMC Psychiatry 2015; 15: 74

[5] Murphy SM, Irving CB, Adams CE, Waqar M. Crisis intervention for people with severe mental illnesses. Cochrane Database Syst Rev; DOI: 10.1002/14651858.CD001087.pub5
[6] Paton F, Wright K, Ayre N et al. Improving outcomes for people in mental health crisis: a rapid synthesis of the evidence for available models of care. Health Technol Assess 2016; 20: 1-162

[7] Johnson S, Needle J. Crisis resolution teams: rationale and core model. In: Johnson S, Needls J, Bindman JP, Thornicroft G, eds. Crisis Resolution and Home Treatment in Mental Health. Cambridge: Cambridge University Press; 2008: $67-84$

[8] Lloyd-Evans B, Bond GR, Ruud T et al. Development of a measure of model fidelity for mental health Crisis Resolution Teams. BMC Psychiatry 2016; 16: 427

[9] Organization for Economic Co-operation and Development (OECD). Making Mental Health Count: The Social and Economic Costs of Neglecting Mental Health Care. OECD Health Policy Studies; DOI: 10.1787/9789264208445-en

[10] Familien-Selbsthilfe Psychiatrie, Bundesverband der Angehörigen psychisch Kranker e. V. Was ist zu tun. Agenda 2020 zur Weiterentwicklung der Versorgung psychisch kranker Menschen. 01.01.2013: Im Internet: www.psychiatrie.de/fileadmin/redakteure/bapk/ueber uns/materialien/agenda_2020_2013.pdf (Stand: 6.1.2017) 